\title{
Is there any role for CAMP-CRP in carbon catabolite repression of the Escherichia coli lac operon?
}

\section{Martine Crasnier-Mednansky}

In their review on carbon catabolite repression (CCR) in bacteria, Boris Görke and Jörg Stülke (Carbon catabolite repression in bacteria: many ways to make the most out of nutrients. Nature Rev. Microbiol. 6, 613-624 (2008)) ${ }^{1}$ specifically analysed CCR in Escherichia coli. The authors reported their view that inducer exclusion, by blocking the entry of lactose, is the main contributor to CCR when wild-type $E$. coli strains are growing in the presence of both glucose and lactose. Under such growth conditions, E. coli strains generally exhibit diauxic growth, which reflects the preferential use of glucose over lactose and the effect of glucose in preventing the use of lactose. The well established 'classical' interpretation of the glucoselactose diauxie is that both inducer exclusion and the transcriptional positive regulation of the lac operon by the cyclic AMP-CRP complex (cAMP-CRP) are necessary for full manifestation of diauxie, as first defined by Jacques Monod ${ }^{2}$ : two distinct exponential growth phases are separated by a complete cessation of growth (lag phase).

The contention that inducer exclusion is the main factor in diauxic growth is supported, in particular, by two articles ${ }^{3,4}$ that oppose the role of cAMP-CRP in the transcriptional regulation of the lac operon in the presence of glucose. Prior to this view, the role of cAMP-CRP in diauxie was deduced based on data which indicated that constitutive $\beta$-galactosidase synthesis (which does not require the inducer for synthesis) was repressed by glucose in the absence of $\mathrm{cAMP}^{5}$ and that the rate of $\beta$-galactosidase synthesis in fully induced cells growing on glucose was less than in cells growing on less-preferred carbon sources ${ }^{6}$. Both observations indicate that glucose transport by the phosphotransferase system (PTS) affect $\beta$-galactosidase synthesis by reducing the level of cAMP. In addition, a typical diauxic lag was eliminated by adding exogenous cAMP to the growth medium ${ }^{5}$.

In support of the view that inducer exclusion is the main factor in diauxie, cAMPCRP was reported to play a crucial part in diauxie only by activating the transcription of the glucose transporter gene ${ }^{4}$. If this were the case, then adding cAMP should have only a limited effect on diauxie or at least should stimulate inducer exclusion by enhancing glucose transport. Consequently, a further decrease in $\beta$-galactosidase expression in the presence of cAMP would result in enhancement of the diauxic lag. In any case, adding cAMP would eliminate the diauxic lag.

The next contentious issue arises from the observation that an E. coli mutant strain that is not sensitive to cAMP (lacL8UV5) exhibits diauxic growth in the presence of glucose and lactose $e^{4}$. Based on this observation, one can conclude, as reported ${ }^{4}$, that the low level of cAMP in cells growing on glucose does not contribute to the manifestation of diauxie. However, it must be recollected that the lacL8UV5 strain of E. coli growing on lactose is impaired in $\beta$-galactosidase synthesis, and has a production rate of $60 \%$ of a wild-type strain ${ }^{7}$. This is the most likely explanation, besides inducer exclusion, for the diauxic growth of strain lacL8UV5. Indeed, the time necessary for synthesizing a sufficient amount of $\beta$-galactosidase (for lactose utilization) will undoubtedly be longer than with a wild-type strain. It thus cannot be inferred that cAMP in a wild-type strain is irrelevant to diauxie, because a cAMPinsensitive mutant strain that is impaired in its ability to synthesize $\beta$-galactosidase exhibits diauxie. Similarly, it should not be concluded, as it has been before ${ }^{3}$, that expression of $\beta$-galactosidase is reduced by glucose solely owing to inducer exclusion in a specific mutant strain that lacks adenylate cyclase and possesses a cAMP-insensitive CRP $\left(c r p^{*}\right)$. Indeed, in such a mutant strain, expression of $\mathrm{CRP}^{\star}$ (encoded by $\left.c r p^{\star}\right)$ is downregulated in the presence of glucose owing to an auto-regulatory circuit that affects the transcription of $c r p^{*}$ (REF. 8). Consequently, in the presence of glucose, the amount of CRP ${ }^{*}$ is reduced compared with the amount of CRP in a wild-type strain. Therefore, besides inducer exclusion, expression of $\beta$-galactosidase is further reduced by a limited amount of CRP $\left(\mathrm{CRP}^{\star}\right)$, which in diauxie affects the transition between growth phases.

Another concern is the amount of cAMP in cells growing on lactose compared with glucose. It has been established that the level of cAMP in lactose-grown cells is low compared with other less-preferred carbon sources, but nevertheless is slightly higher than the level of cAMP in glucose-grown cells ${ }^{9}$. Indeed, phosphorylated enzyme IIA $^{\text {glc }}$ (which activates adenylate cyclase) is detected in lactose-grown cells but not in glucose-grown cells, as supported by the phosphorylation state of enzyme IIA glc detected by Western blotting ${ }^{10}$.

Finally, other data suggest a role for cAMP-CRP in diauxie. For example, a PTS sugar-like mannitol can substitute for glucose in diauxie production in E. coli whereas others, such as fructose, do not ${ }^{2}$. This correlates with the observation that the level of cAMP in mannitol-grown cells is in the same range as in glucose-grown cells, although the levels of cAMP in both mannitol-grown and glucose-grown cells are lower than in fructose-grown cells ${ }^{11}$.

Owing to the factors discussed above, transcriptional regulation cannot be ruled out as a mechanism that supports diauxic growth in E. coli. However, it should be emphasized that inducer exclusion (which has received less attention than transcriptional regulation) is also a major factor in diauxie and CCR in general. Undoubtedly, bacteria have developed multiple mechanisms to assure the preferential use of a carbon source, thereby allowing fast growth.

Martine Crasnier-Mednansky is at the Mednansky Institute, PO Box 940, Pine Valley, California 91962, USA

e-mail: martine@minst.org

Görke, B. \& Stülke, J. Carbon catabolite repression in bacteria: many ways to make the most out of nutrients. Nature Rev. Microbiol. 6, 613-624 (2008).

2 Monod, J. Recherches sur la Croissance des Cultures Bactériennes. Thesis, Hermann et Cie, Paris (1942).

3. Inada, T., Kimata, K. \& Aiba, H. Mechanism responsible for glucose-lactose diauxie in Escherichia coli: challenge to the cAMP model. Genes Cells 1, 293-301 (1996).

4. Kimata, K., Takahashi, H., Inada, T., Postma, P. \& Aiba, H. cAMP receptor protein-cAMP plays a crucial role in glucose-lactose diauxie by activating the major glucose transporter gene in Escherichia coli. Proc. Natl Acad. Sci. USA 94, 12914-12919 (1997).

5. Ullmann, A. \& Monod, J. Cyclic AMP as an antagonist of catabolite repression in Escherichia coli. FEBS Lett. 2 , 57-60 (1968)

6. Pastan, I. \& Perlman, R. Cyclic adenosine monophosphate in bacteria. Science 169, 339-344 (1970).

Silverstone, A. E., Arditti, R. R. \& Magasanik, B. Catabolite-insensitive revertants of lac promoter mutants. Proc. Natl Acad. Sci. USA 66, 773-779 (1970).

8. Tagami, H., Inada, T., Kunimura, T. \& Aiba, H. Glucose lowers CRP* levels resulting in repression of the lac operon in cells lacking cAMP. Mol. Microbiol. 17 , 251-258 (1995).

9. Crasnier, M. \& Danchin, A. Characterization of Escherichia coli adenylate cyclase mutants with modified regulation. J. Gen. Microbiol. 136, 1825-1831 (1990).

10. Hogema, B. M. et al. Inducer exclusion in Escherichia coli by non-PTS substrates: the role of the PEP to pyruvate ratio in determining the phosphorylation state of enzyme IIA ${ }^{\mathrm{Gl}}$. Mol. Microbiol. 30, 487-498 (1998).

1. Epstein, W., Rothman-Denes, L. B. \& Hesse, J. Adenosine 3':5'-cyclic monophosphate as mediator of catabolite repression in Escherichia coli. Proc. Nat Acad. Sci. USA 72, 2300-2304 (1975). 